\title{
Melting of a 2D quantum electron solid in high magnetic field
}

\author{
YONG P. CHEN ${ }^{1,2 \star \S}$, G. SAMBANDAMURTHY ${ }^{1,2}$, Z. H. WANG ${ }^{2,3}$, R. M. LEWIS ${ }^{1,2 \dagger}$, L. W. ENGEL 2 , \\ D. C. TSUI ${ }^{1}$, P. D. YE ${ }^{1,2 \ddagger}$, L. N. PFEIFFER ${ }^{4}$ AND K. W. WEST ${ }^{4}$ \\ ${ }^{1}$ Department of Electrical Engineering, Princeton University, Princeton, New Jersey 08544, USA \\ ${ }^{2}$ National High Magnetic Field Laboratory, Tallahassee, Florida 32310, USA \\ ${ }^{3}$ Department of Physics, Princeton University, Princeton, New Jersey 08544, USA \\ ${ }^{4}$ Bell Laboratories, Murray Hill, New Jersey 07974, USA \\ *Current address: The Richard E. Smalley Institute for Nanoscale Science and Technology and Department of Physics, Rice University, Houston, Texas 77005, USA \\ †Current address: Department of Physics, University of Maryland, College Park, Maryland 20742, USA \\ ${ }^{\ddagger}$ Current address: School of Electrical and Computer Engineering, Purdue University, West Lafayette, Indiana 47907, USA \\ ¿e-mail: yongchen@rice.edu
}

T The melting temperature $T_{\mathrm{m}}$ of a solid is generally determined by its solid-liquid transition on being heated at a fixed pressure, usually ambient pressure. It is also determined indirectly by the density $\boldsymbol{n}$ by means of the equation of state. This remains true even for solid helium ${ }^{1}$, in which quantum effects often lead to unusual properties ${ }^{2}$. Here, we present experimental evidence to show that for a two-dimensional (2D) solid formed by electrons in a semiconductor sample under a strong perpendicular magnetic field ${ }^{3}(B), T_{\mathrm{m}}$ is not controlled by $n$, but effectively by the quantum correlation between the electrons through the Landau level filling factor $v=n h / e B$ (where $h$ is the Planck constant and $e$ is the electronic charge). Such melting behaviour, different from that of all other known solids (including a classical 2D electron solid at zero magnetic field ${ }^{4}$ ), suggests the quantum nature of the magnetic-field-induced electron solid. Moreover, $T_{\mathrm{m}}$ increases with the strength of the sample-dependent disorder that tends to pin the electron solid in place.

Electrons are expected to crystallize into a solid (so-called 'Wigner crystal' ${ }^{5}$ ) when the (Coulomb) interaction energy between the electrons sufficiently dominates over the kinetic energy. One example of such an electron solid was found in a very low-density two-dimensional electron system (2DES) realized on helium surfaces $^{4}$ (at zero magnetic field). Because of the low $n$, the zero-point motion (given by the Fermi energy $E_{\mathrm{f}}=n h^{2} / 2 \pi m$, where $m$ is the electron mass) is negligibly small and at finite temperatures $(T)$, as in the experiment ${ }^{4}$, the kinetic energy originates mainly from the classical thermal motion $\left(k_{\mathrm{B}} T\right)$. The melting of such a 'classical' $2 \mathrm{D}$ electron solid is determined only by the competition between the thermal kinetic energy and Coulomb interaction $\left(e^{2} \sqrt{n} / 4 \pi \epsilon\right.$, where $\epsilon$ is the dielectric constant) (the International System of Units, SI, is used exclusively in this paper) and is thought to be describable by the Kosterlitz-Thouless theory of 2D melting ${ }^{6}$. Experimentally, the melting was found to occur ${ }^{4}$ at $T_{\mathrm{cm}}=e^{2} \sqrt{n} /\left(4 \pi \epsilon k_{\mathrm{B}} \Gamma\right)$ (where $k_{\mathrm{B}}$ is the Boltzman constant) with $\Gamma \sim 130$, in excellent agreement with theoretical calculations $s^{7,8}$.

The 2DES as realized in the high-quality GaAs/AlGaAs structures in our experiment has relatively high $n$, thus (in the absence of magnetic fields) the zero-point motion $\left(E_{\mathrm{f}}\right)$ is significant and the 2DES does not solidify even at $T=0$. However, it is well known that a sufficiently strong perpendicular magnetic field $(B)$ tends to suppress the kinetic energy of $2 \mathrm{D}$ electrons and induce the solidification ${ }^{9,10}$. On the other hand, at finite $B$ the motion of electrons is quantized into Landau levels, and delicate many-body quantum correlation $s^{11,12}$ among electrons can cause the $2 \mathrm{DES}$ to condense into fractional quantum Hall $(\mathrm{FQH})^{13}$ liquid states at certain rational fractional values of Landau level filling factor $v=n h / e B$. Experimentally, the magnetic-field-induced electron solid (MIES) ${ }^{3}$ forms at sufficiently small $v$, following the termination of $\mathrm{FQH}$ states at low $T$. It is deformed and pinned by disorder in the system, thus it behaves as an insulator (in d.c. transport), and has a characteristic resonance (see, for example, ref. 14) in its frequency $(f)$ dependent, real diagonal conductivity $\left(\operatorname{Re}\left[\sigma_{x x}(f)\right]\right)$ due to the 'pinning mode' of domains of the elastic solid oscillating collectively around the disorder ${ }^{15-17}$.

The melting of such a MIES has been studied in various experiments ${ }^{18-23}$, and it was commonly presumed ${ }^{8,18,20,22}$ that at a fixed $v$ the melting should be similar to that of a classical electron solid (the expected exact ground state for a 2DES at infinite $B$ ), and thus $T_{\mathrm{m}}$ would be determined by $n$, as $T_{\mathrm{cm}}$ is. In this letter, we show unambiguously that this is not the case, that is, the melting of a MIES does not behave as a classical electron solid, and in fact in any given sample (whose intrinsic disorder is fixed), $T_{\mathrm{m}}$ is determined by $v$, not $n$, and is unrelated to $T_{\mathrm{cm}}$.

In our experiments we have studied the $T$-dependence of the pinning mode resonance of the MIES in its $\operatorname{Re}\left[\sigma_{x x}(f)\right]$ spectrum 

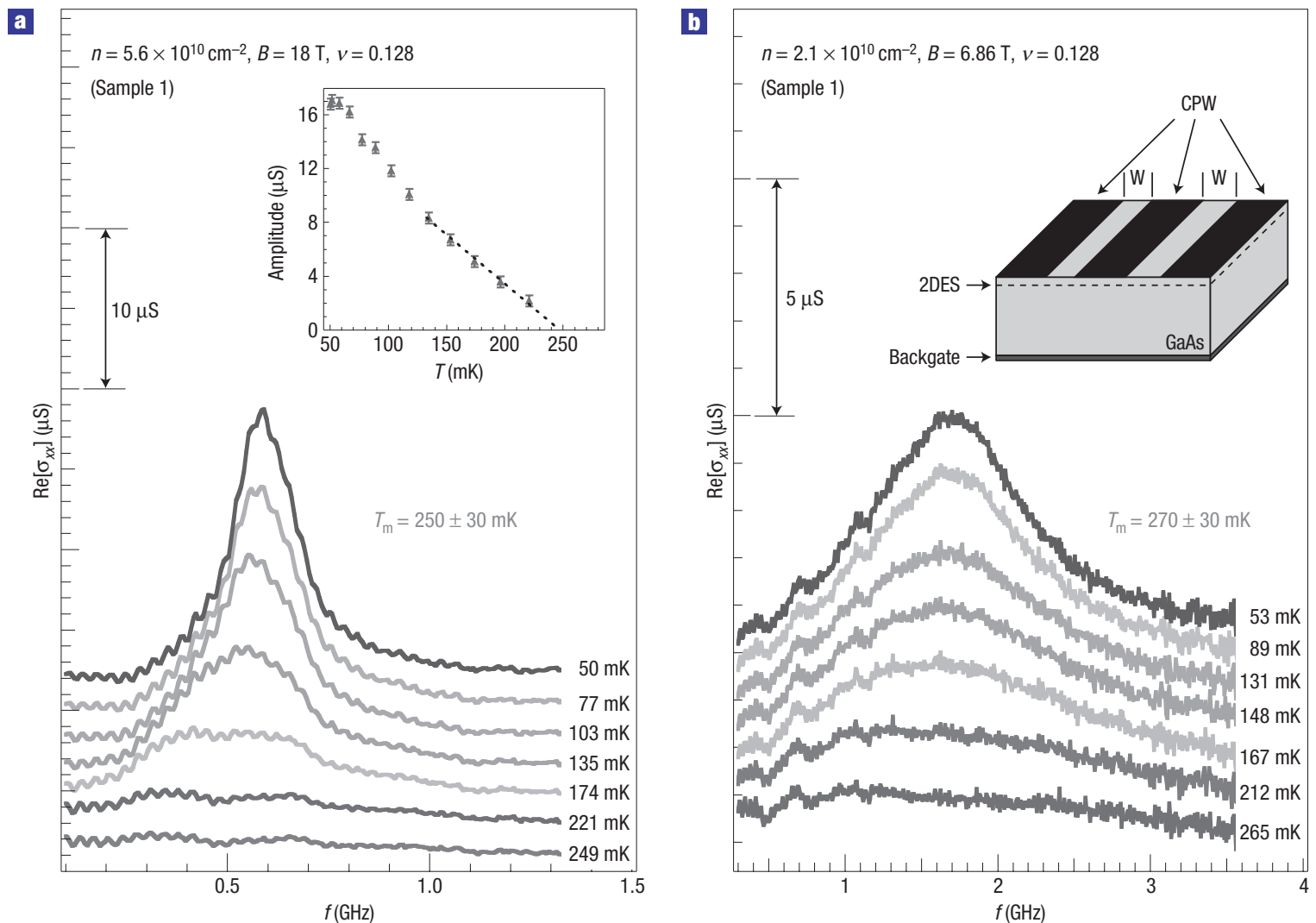

Figure 1 Temperature $(T)$ dependence of the microwave spectra of the 2D electron solid measured from sample 1 at two different densities $(n)$ with the same Landau filling factor $(v=n h / e B)$. a, $T$-dependence of the spectra at $n=5.6 \times 10^{10} \mathrm{~cm}^{-2}$ with $v=0.128$ (magnetic field $B=18 \mathrm{~T}$ ). Spectra at a series of representative $T$ values are shown and offset for clarity. The pinning resonance of the electron solid observed at $T=50 \mathrm{mK}$ is seen to weaken with increasing $T$ and disappear at $\sim 250 \mathrm{mK}$, taken as the melting temperature $\left(T_{\mathrm{m}}\right)$ of the electron solid. The inset shows that the amplitude of the resonance extrapolates to zero at the similar $T_{\mathrm{m}}$. Typical error bars for the resonance amplitude are less than $0.5 \mu \mathrm{S}$. b, $T$-dependence of the spectra at $n=2.1 \times 10^{10} \mathrm{~cm}^{-2}$ with $v=0.128(B=6.86 \mathrm{~T})$. The low $T$ resonance disappears at a $T_{\mathrm{m}}$ similar to that shown in $\mathbf{a}\left(n=5.6 \times 10^{10} \mathrm{~cm}^{-2}\right)$, despite the fact that $n$ has been reduced by more than a factor of two here (using a negative voltage between a backgate and the $2 \mathrm{DES}$ ). The inset shows a schematic diagram of the sample. The dark regions on the top surface represent the coplanar waveguide (CPW). The backgate allows the in situ change of $n$.

measured by microwave spectroscopy ${ }^{24}$. No resonance is observed when $T$ is raised above some characteristic $T_{\mathrm{m}}$, taken as the melting $T$ of the electron solid. By systematically measuring $T_{\mathrm{m}}$ while varying both $n$ and $B$, we found that within the experimental resolution, $T_{\mathrm{m}}$ in a given sample is only a function of $v$ (that is, $\left.T_{\mathrm{m}}(n, B)=T_{\mathrm{m}}(n / B)\right)$ down to the smallest $v \sim 0.05$ attained in our experiments. Although $T_{\mathrm{m}}$ is generally sensitive to $n$ at fixed $B$, $T_{\mathrm{m}}$ is insensitive to $n$ at fixed $v$. As $v=n h / e B=2\left(l_{\mathrm{B}} / r\right)^{2}$, where the magnetic length $l_{\mathrm{B}}=\sqrt{\hbar / e B}$ (where $\hbar=h / 2 \pi$ ) is a measure of the size of the single electron wavefunction and $r=1 / \sqrt{\pi n}$ is the mean separation between the electrons, our findings reflect the quantum nature of the 2D electron solid formed at finite $B$ and demonstrate that its melting $\left(T_{\mathrm{m}}\right)$ is effectively controlled by the inter-electron quantum correlation, which depends on $l_{\mathrm{B}} / r$.

We have studied two different 2DES samples. Sample 1 is a GaAs/AlGaAs heterojunction. Sample 2 is a 15 -nm-wide $\mathrm{AlGaAs} / \mathrm{GaAs} / \mathrm{AlGaAs}$ quantum well $(\mathrm{QW})$. By backgating and/or having different cooldowns, the electron densities in both samples can be tuned to various extents (to be specified below). Typically at their respective as-cooled $n$, sample 1 has mobility $\mu \sim 6 \times$ $10^{6} \mathrm{~cm}^{2} \mathrm{~V}^{-1} \mathrm{~s}^{-1}$, and sample 2 has $\mu \sim 1 \times 10^{6} \mathrm{~cm}^{2} \mathrm{~V}^{-1} \mathrm{~s}^{-1}$.

The inset of Fig. $1 \mathrm{~b}$ shows a schematic local cross-section of a typical sample (not to scale). A metal film coplanar waveguide (CPW), lithographically deposited on the surface, enables the measurement of $\operatorname{Re}\left[\sigma_{x x}(f)\right]$ of the 2DES. A network analyser generates a microwave signal propagating along the CPW and coupling capacitively to the 2DES (located $0.1-0.5 \mu \mathrm{m}$ below the surface). The measured relative power absorption $(P)$ by the $2 \mathrm{DES}$ can be related ${ }^{24}$ to $\operatorname{Re}\left[\sigma_{x x}\right]$ of the $2 \mathrm{DES}$ as $P=\exp \left(\left(2 l Z_{0} / w\right) \operatorname{Re}\left[\sigma_{x x}\right]\right)$, where $l$ and $w$ are the total length and slot width of the CPW respectively and $Z_{0}=50 \Omega$ is the CPW characteristic impedance. CPW of meander shapes ${ }^{24}$ are commonly used to obtain larger geometric ratios $(2 l / w)$, and therefore to increase the strength of the signal $(P)$. The sample is mounted on a metal block that is kept in good thermal equilibrium with the mixing chamber of a dilution refrigerator during the measurements. The microwave input is kept in the low power limit by reducing the power until the signal $(P)$ no longer changes. A negative voltage between a backgate (located $\sim 200 \mu \mathrm{m}$ below the surface) and the 2DES enables in situ reduction of $n$ from the as-cooled values.

Figure 1 shows the $T$-dependence of the microwave resonance of the electron solid in sample 1 and the determination of $T_{\mathrm{m}}$ at two different values of $n$ with $v$ fixed at a representative value of 0.128. In Fig. 1a, $n=5.6 \times 10^{10} \mathrm{~cm}^{-2}(B=18 \mathrm{~T})$ and the $\operatorname{Re}\left[\sigma_{x x}(f)\right]$ spectrum shows a clear resonance with peak frequency $\left(f_{\mathrm{pk}}\right)$ near $600 \mathrm{MHz}$ at low $T(\sim 50 \mathrm{mK})$. As $T$ is increased, the resonance weakens. Its $f_{\mathrm{pk}}$ at elevated $T$ also decreases slightly from the base $T$ 


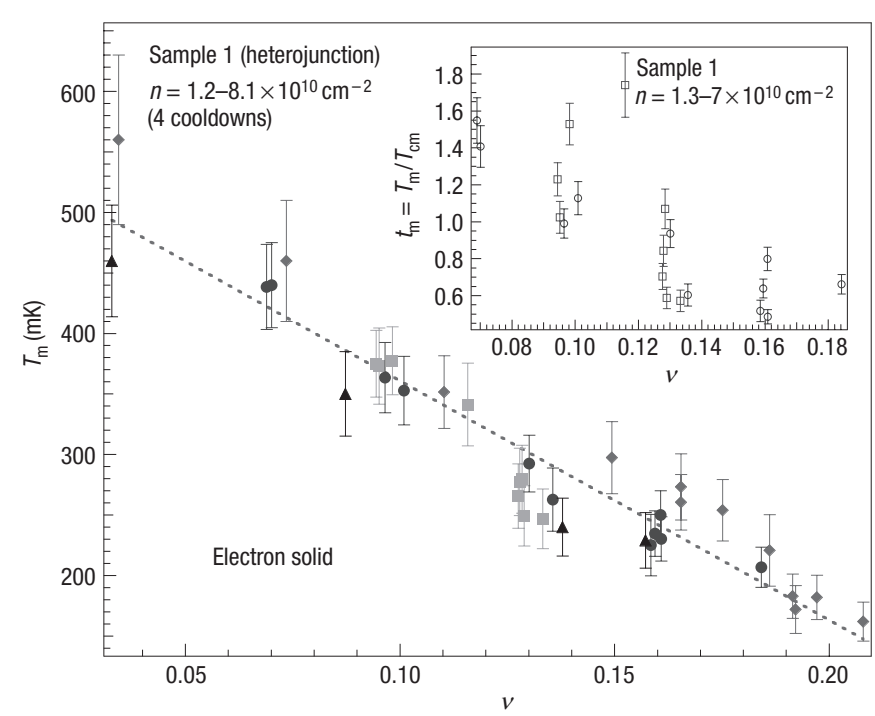

Figure $2\left(T_{\mathrm{m}}, v\right)$ phase diagram for the electron solid in sample 1. The $T_{\mathrm{m}}$ values are measured in a total of four cooldowns (shown as diamonds, circles, squares and triangles), over a wide range of densities $\left(n=1.2-8.1 \times 10^{10} \mathrm{~cm}^{-2}\right)$ and magnetic fields. Within the experimental uncertainty, $T_{\mathrm{m}}$ versus $v$ gives rise to a well-defined melting curve of the electron solid. The dashed line is a guide to the eye, obtained by a linear fit through all of the data. Typical error bars in $T_{\mathrm{m}}$ are less than $10 \%$. The inset shows the 'reduced' $t_{\mathrm{m}}$ versus $v$ from two cooldowns. $t_{\mathrm{m}}$ is defined as $T_{\mathrm{m}}$ normalized by the classical 2D electron-solid melting temperature

$T_{\mathrm{cm}}=e^{2} \sqrt{n} /\left(4 \pi \epsilon k_{\mathrm{B}} \Gamma\right)$ (where we take $\left.\Gamma=127\right) . t_{\mathrm{m}}$ versus $v$ does not result in a well-defined melting curve, indicating that the melting temperature $T_{\mathrm{m}}$ is not determined by $n$ or $T_{\mathrm{cm}}$.

( $50 \mathrm{mK}$ ) value, but by no more than $20 \%$, indicating that there is no significant loss of pinning of the electron solid due to the increase in the temperature. The resonance disappears into noise background at $\sim 250 \mathrm{mK}$, which we take as the melting $T\left(T_{\mathrm{m}}\right)$ of the electron solid. The inset of Fig. 1a shows that the resonance amplitude (obtained from a lorentzian fit) extrapolates to zero at a similar $T_{\mathrm{m}}$. In Fig. $1 \mathrm{~b}, n$ has been reduced to $2.1 \times 10^{10} \mathrm{~cm}^{-2}$ (using a backgate voltage of $-300 \mathrm{~V}$ ) while $B$ is also reduced to $6.86 \mathrm{~T}$ to keep $v$ the same value as in Fig. 1a. The lower $n$ reduces the electron-electron interaction relative to the electron-disorder interaction, and the pinning resonance of the electron solid now occurs (at low $T$ ) near $1.6 \mathrm{GHz}$, which is significantly higher than that in Fig. 1a. However, the resonance disappears above a $T_{\mathrm{m}} \sim 270 \mathrm{mK}$, which is similar to the $T_{\mathrm{m}}$ in Fig. 1a, within the experimental uncertainty in determining $T_{\mathrm{m}}$ (typically $\sim 10 \%$ ).

We have measured $T_{\mathrm{m}}$ in four different cooldowns of sample 1 for many combinations of $n$ and $B$, at which a resonance from the MIES can be detected. We plot all of these $T_{\mathrm{m}}$ data as a function of $v$ in Fig. 2. Sample 1 has a particularly large range of tunable $n$, from $\sim 1.2-8.1 \times 10^{10} \mathrm{~cm}^{-2}$, covering a $v$ range from $\sim 0.21$ down to $\sim 0.03$. Generally, we found $T_{\mathrm{m}}$ to be sensitive to $n$ or $B$ separately (when fixing one and changing the other). On the other hand, near similar $v$ (changing both $n$ and $B$ while fixing $n / B$ ), we have always found $T_{\mathrm{m}}$ to be insensitive to $n$ (or $B$ ), within the experimental errors in $T_{\mathrm{m}}$. Different cooldowns can vary $T_{\mathrm{m}}$ by up to $\sim 15 \%$ (at similar $v$ ) but this does not affect our conclusion. Thus, we find $T_{\mathrm{m}}$ to be mainly determined by $v$, so that $T_{\mathrm{m}}$ versus $v$ as plotted in Fig. 2 defines a melting curve for the electron solid in sample 1. A linear fit of $T_{\mathrm{m}}$ versus $v$ gives a guide to the eye, shown as the dashed line, which lies within $20 \%$ of all of the $\left(T_{\mathrm{m}}, v\right)$ data points and within $10 \%$ of a majority $(70 \%)$ of them.

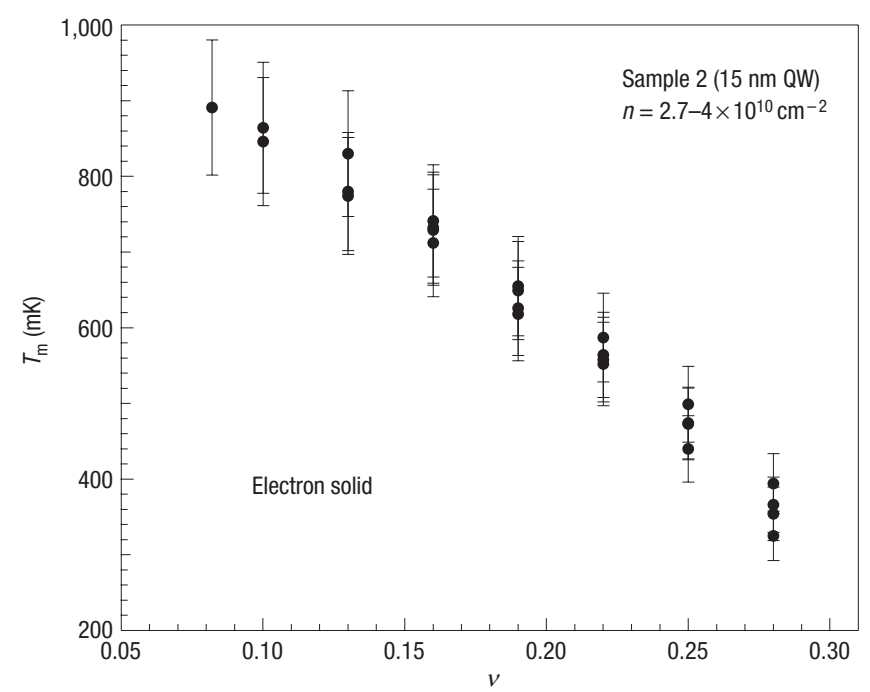

Figure $3\left(T_{\mathrm{m}}, v\right)$ melting curve of the electron solid in sample 2, a narrow QW of width $15 \mathrm{~nm}$. Sample 2 has a tunable $n=2.7-4 \times 10^{10} \mathrm{~cm}^{-2}$ and enters an electron-solid phase at $v<0.3$. Typical error bars in $T_{\mathrm{m}}$ are $10 \%$.

In the inset of Fig. 2 we plot the 'reduced's,18 $t_{\mathrm{m}}=T_{\mathrm{m}} / T_{\mathrm{cm}}$ from two similar cooldowns versus $v$, where $T_{\mathrm{cm}}=e^{2} \sqrt{n} /\left(4 \pi \epsilon k_{\mathrm{B}} \Gamma\right)$ is the melting $T$ of a classical 2D electron solid defined earlier (with the value $\left.{ }^{7,8} \Gamma=127\right)$. In contrast to $T_{\mathrm{m}}, t_{\mathrm{m}}$ can vary significantly (sometimes by a factor of 3) at similar $v$. Thus, $t_{\mathrm{m}}$ versus $v$ does not give a well-defined melting curve for the electron solid, confirming that $T_{\mathrm{m}}$ is not determined by $n$ or $T_{\mathrm{cm}}$. We have also checked that neither $T_{\mathrm{m}}$ nor $t_{\mathrm{m}}$ plotted against either $n$ or $B$ gives any well-defined melting curve. Furthermore, we notice that our measured $T_{\mathrm{m}}$ is also unrelated to the hypothetical 'thermal depinning' temperature $\left(T_{\text {depin }}\right)$ of domains ${ }^{15-17}$ of the electron crystal. In fact, we can estimate $T_{\text {depin }} \sim\left(1 / k_{\mathrm{B}}\right) m_{\mathrm{e}} \omega_{0}^{2} \xi^{2}(L / a)^{2} \propto n^{3 / 2}$ (where $\omega_{0}^{2}=2 \pi f_{\mathrm{pk}} e B / m_{\mathrm{e}} \propto n^{-3 / 2}$ characterizes the 'effective pinning potential'16 (we note that $\omega_{0}$ is also much larger than $k_{\mathrm{B}} T_{\mathrm{m}} / \hbar$ ), $\xi$ is the disorder correlation length, $L \propto n$ is the Larkin domain size ${ }^{15-17}$ and $a$ is the electron crystal lattice constant), in contrast to $T_{\mathrm{m}}$, which is determined by $v$, and as seen in Fig. 2, typically decreases with increasing $n$ if $B$ is fixed.

Figure 3 shows the $\left(T_{\mathrm{m}}, v\right)$ melting curve measured on sample 2 (15-nm-wide QW). Sample 2 has a tunable $n=2.7-4 \times 10^{10} \mathrm{~cm}^{-2}$. Probably owing to the relatively narrow confinement of the 2DES in the QW, sample 2 enters the solid phase for $v<0.3$ and the typical $f_{\mathrm{pk}}$ observed is $\sim 6-8 \mathrm{GHz}$. In this sample we again found $T_{\mathrm{m}}$ to be controlled by $v$, although the value of $T_{\mathrm{m}}$ is higher than that in sample 1 at similar $v$.

In each of the two samples, at a fixed high $B, T_{\mathrm{m}}$ typically increases with decreasing $n$. This is opposite to the classical behaviour, where $T_{\mathrm{cm}}$ decreases with decreasing $n$. At a fixed $v$, our observed $T_{\mathrm{m}}$ is insensitive to $n$, and $T_{\mathrm{m}}$ in each sample is a well-defined function of $v$. Recent theories ${ }^{25,26}$ have suggested that many-body quantum correlations between electrons can still be important in the solid phase terminating the FQH states at high $B$. The parameter that captures such quantum correlations is the Laudau filling factor $v=2\left(l_{\mathrm{B}} / r\right)^{2}$, which also determines the (single-particle) wavefunction overlap ${ }^{27}$ between neighbouring electrons. This overlap $\left(I_{\mathrm{s}}\right)$, given ${ }^{27}$ by $\mathrm{e}^{-(2 / \sqrt{3}) \pi / \nu}$, is in fact quite small in the high- $B$, low- $v$ regime of the MIES. Our data show that $T_{\mathrm{m}}$ is quite strongly dependent on $v$ (for example, $T_{\mathrm{m}}$ changes 
by almost a factor of 2 when $v$ is changed by a factor of 2). Therefore the dependence of $T_{\mathrm{m}}$ on $v$ is unlikely to result from a $T_{\mathrm{m}}$ that is determined by the Coulomb Hartree energy $E_{\mathrm{H}}$ (which is shown ${ }^{27}$ to have very weak $v$-dependence in this low- $v$ regime, where the wavefunction overlap $I_{\mathrm{s}}$ has a negligible contribution to $\left.E_{\mathrm{H}}\right)$. Our findings indicate that the melting of MIES is effectively controlled by the inter-electron quantum correlation ${ }^{27}$, through $v$. The well-defined $\left(T_{\mathrm{m}}, v\right)$ melting curve we obtained constitutes the phase boundary between a quantum solid and a correlated quantum liquid ${ }^{17}$ in each sample.

The $T_{\mathrm{m}}$ we measured in both samples are of a similar order of magnitude to those measured previously in other samples with various experimental techniques ${ }^{18-23}$. We have noticed that at similar $v$, sample 2 (narrow $\mathrm{QW}$ ) has a higher $T_{\mathrm{m}}$ than sample 1 (heterojunction). It has been suggested ${ }^{15}$ that the relevant disorder in semiconductor samples that pins the MIES comes mostly from the interfaces vertically confining the 2DES (this is consistent with the relatively high $f_{\mathrm{pk}}$ observed in the narrow QW sample). Thus, our findings suggest that a $2 \mathrm{D}$ electron solid subjected to stronger pinning disorder melts at higher $T_{\mathrm{m}}$, an effect that has been predicted previously ${ }^{28}$. Interestingly, we notice that studies on the effects of pinning disorder or geometric confinement ${ }^{29}$ on $T_{\mathrm{m}}$ in other solids usually find the opposite behaviour. For example, both the vortex solid in a high-temperature superconductor subjected to artificial pinning centres ${ }^{30}$ and a helium solid in a porous glass ${ }^{31}$ show a depression of $T_{\mathrm{m}}$ with the added disorder. Disorder is generally unavoidable in semiconductor samples, and as seen here can strongly influence the values of $T_{\mathrm{m}}$ measured, which may not be those of an ideal, clean Wigner crystal. On the other hand, the observation that in any given sample (thus with fixed intrinsic disorder), $T_{\mathrm{m}}$ is not sensitive to $n$ (nor to $B$ ) if $v=n h / e B$ is fixed (but is sensitive to $n$ if $B$ is fixed) is difficult to explain in a classical picture of, for example, interplay of disorder and $n$ (screening). The fact that $v$ is found to be the controlling variable for $T_{\mathrm{m}}$ in samples of quite different disorder (including sample 1, in which it can be inferred from the observed pinning resonance that the Wigner crystal there can possess substantial correlation length ${ }^{14-17}$ ) suggests that such a behaviour ( $T_{\mathrm{m}}$ being controlled by $v$ ) arises from some mechanism in which many-body quantum correlation between electrons may be important. The exact role of disorder (perhaps involving its interplay with quantum correlation) in determining the value and behaviour of $T_{\mathrm{m}}$ remains to be better understood.

It is also interesting to compare the quantum nature of our $2 \mathrm{D}$ electron solid with the quantum solids of helium. In a helium solid, the size of atoms is fixed by nature and the quantum parameter is the De Boer parameter ${ }^{1} \Lambda \sim h /(a \sqrt{M v})$ (where $M$ is the atomic mass, $a$ the inter-atomic distance and $v$ the inter-atomic potential strength), which is fixed at fixed $n$. The $T_{\mathrm{m}}$ of a helium solid is only determined by $n$. In the case of a $2 \mathrm{D}$ electron solid formed in high $B$, the size of the single electron wavefunction $\left(l_{\mathrm{B}}\right)$ is readily tunable by $B$, independently of $n$. The quantum parameter here is $v$ $=n h / e B$, and we have found that $T_{\mathrm{m}}$ of such an electron solid in a given sample (with fixed disorder) is controlled by $v$ rather than $n$.
Received 28 December 2005; accepted 5 May 2006; published 4 June 2006.

\section{References}

1. Wilks, J. The Properties of Liquid and Solid Helium (Oxford Univ. Press, Oxford, 1967).

2. Kim, E \& Chan, M. H. W. Probable observation of a supersolid helium phase. Nature 427, 225-227 (2004).

3. Shayegan, M. in Perspectives in Quantum Hall Effects (eds Das Sarma, S. \& Pinczuk, A.) Ch. 9 (Wiley, New York, 1997)

4. Grimes, C. C. \& Adams, G. Evidence for a liquid-solid phase transition in a classical, two-dimensional sheet of electrons. Phys. Rev. Lett. 42, 795-798 (1979).

5. Wigner, E. P. On the interaction of electrons in metals. Phys. Rev. 46, 1002-1011 (1934)

6. Thouless, D. Melting of the two-dimensional Wigner lattice. J. Phys. C 11, L189-L190 (1978).

7. Morf, R. H. Temperature dependence of the shear modulus and melting of the two-dimensional electron solid. Phys. Rev. Lett. 43, 931-935 (1979).

8. Chui, S. T. \& Esfariani, K. Finite-temperature two-dimensional Wigner transition. Phys. Rev. B 44, 11498-11501 (1991)

9. Lozovik, Y. E. \& Yudson, V. I. Crystallization of a two-dimensional electron gas in a magnetic field. JETP Lett. 22, 11-12 (1975).

10. Fukuyama, H., Platzman, P. M. \& Anderson, P. W. Two dimensional electron gas in a strong magnetic field. Phys. Rev. B 19, 5211-5217 (1979).

11. Laughlin, R. B. Anomalous quantum hall effect: An incompressible quantum fluid with fractionally charged excitations. Phys. Rev. Lett. 50, 1395-1398 (1983).

12. Kivelson, S., Kallin, C., Arovas, D. P. \& Schrieffer, J. R. Cooperative ring exchange theory and the fractional quantum hall effect. Phys. Rev. B 36, 1620-1646 (1987).

13. Tsui, D. C., Stormer, H. L. \& Gossard, A. C. Two-dimensional magnetotransport in the extreme quantum limit. Phys. Rev. Lett. 48, 1559-1562 (1982).

14. Ye, P. D. et al. Correlation lengths of the Wigner-crystal order in a two-dimensional electron system at high magnetic fields. Phys. Rev. Lett. 89, 176802 (2002).

15. Fertig, H. A. Electromagnetic response of a pinned Wigner crystal. Phys. Rev. B 59, 2120-2141 (1999). 16. Fogler, M. M. \& Huse, D. A. Dynamical response of a pinned two-dimensional Wigner crystal. Phys. Rev, B 62, 7553-7570 (2000).

17. Chitra, R., Giamarchi, T. \& Le Doussal, P. Pinned Wigner crystals. Phys. Rev. B 65, 035312 (2001).

18. Glattli, D. C. et al. Experiments on ordering in classical and quantum 2 d electron systems. Surf. Sci. 229, 344-351 (1990).

19. Goldman, V. J., Santos, M., Shayegan, M. \& Cunningham, J. E. Evidence for two-dimensional quantum Wigner crystal. Phys. Rev. Lett. 65, 2189-2192 (1990).

20. Williams, F. I. B. et al. Conduction threshold and pinning frequency of magnetically induced Wigner solid. Phys. Rev. Lett. 66, 3285-3288 (1991).

21. Paalanen, M. A. et al. Electrical conductivity and Wigner crystallization. Phys. Rev. B 45, 13784-13787 (1992).

22. Goldys, E. M. et al. Magneto-optical probe of two-dimensional electron liquid and solid phases. Phys. Rev. B 46, 7957-7960 (1992).

23. Kukushkin, I. V. et al. Wigner solid vs. incompressible Laughlin liquid: phase diagram derived from time-resolved photoluminescence. Europhys. Lett. 23, 211-216 (1993).

24. Engel, L.W., Shahar, D., Kurdak, C.. \& Tsui, D. C. Microwave frequency dependence of integer quantum hall effect: Evidence for finite-frequency scaling. Phys. Rev. Lett. 71, 2638-2641 (1993).

25. Yi, H. \& Fertig, H. A. Laughlin-Jastrow-correlated Wigner crystal in a strong magnetic field. Phys. Rev. B 58, 4019-4027 (1998).

26. Chang, C. C., Jeon, G. S. \& Jain, J. K. Microscopic verification of topological electron-vortex binding in the lowest Landau-level crystal state. Phys. Rev. Lett. 94, 016809 (2005).

27. Maki, K. \& Zotos, X. Static and dynamic properties of a two-dimensional Wigner crystal in a strong magnetic field. Phys. Rev. B 28, 4349-4356 (1983).

28. Tsukada, M. Two-dimensional crystallization of the electrons in MOS structures induced by strong magnetic field. J. Phys. Soc. Japan 42, 391-398 (1977).

29. Christensen, H. O. Confinement effects on freezing and melting. J. Phys. Condens. Matter 13, R95-R133 (2001).

30. Paulius, L. M. et al. Evolution of the vortex phase diagram in $\mathrm{YBa}_{2} \mathrm{Cu}_{3} \mathrm{O}_{7-\delta}$ with random point disorder. Phys. Rev. B 61, R11910-R11913 (2000).

31. Beamish, J. R., Hikata, A., Tell, L. \& Elbaum, C. Solidification and superfluidity of ${ }^{4} \mathrm{He}$ in porous vycor glass. Phys. Rev. Lett. 50, 425-428 (1983).

\section{Acknowledgements}

Financial support for this work was provided by DOE grant No. DE-FG02-05ER46212, the NHMFL in-house research program, and AFOSR. The spectroscopy measurements were carried out at the National High Magnetic Field Laboratory, which is supported by NSF Cooperative Agreement No. DMR-0084173 and by the State of Florida. We thank G. Jones, T. Murphy and E. Palm at NHMFL for experimental assistance. We also thank S. T. Chui, R. L. Willett, K. Yang and C. C. Yu for inspirational discussions, and thank H. A. Fertig and P. B. Littlewood in particular for discussions regarding the thermal depinning temperature of the Wigner crystal.

Correspondence and requests for materials should be addressed to Y.P.C.

Competing financial interests

The authors declare that they have no competing financial interests.

Reprints and permission information is available online at http://npg.nature.com/reprintsandpermissions/ 